\title{
Article
}

\section{DNA environment of centromeres and non-homologous chro- mosomes interactions in mouse}

\author{
Victor Spangenberg ${ }^{1 *}$, Mikhail Losev ${ }^{1}$, Ilya Volkhin ${ }^{1}$, Svetlana Smirnova1 ${ }^{1}$ Pavel Nikitin ${ }^{1}$ and Oxana Kolomiets $^{1}$ \\ 1 Vavilov Institute of General Genetics, Russian Academy of Sciences, Moscow, Russia \\ * Correspondence: vspangenberg@gmail.com; spangenberg@vigg.ru
}

\begin{abstract}
Pericentromeric regions of chromosomes enriched in tandemly repeated satellite DNA although representing a significant part of eukaryotic genomes are still understudied mainly due to interdisciplinary knowledge gaps. Recent studies suggest their important role in genome regulation, karyotype stability and evolution. Thus, the idea of satellite DNA as a junk part of the genome was refuted. Integration of data about molecular composition, chromosome behaviour and details of in situ organization of pericentromeric regions is of great interest. The objective of this work was a cytogenetic analysis of the interactions of pericentromeric regions non-homologous chromosomes in mouse spermatocytes using immuno-FISH. We analysed two events: the associations between cerntomeric regions of $\mathrm{X}$ chromosome and autosomes, and associations between centromeric regions of autosomal bivalents forming chromocenters.

We conclude that $\mathrm{X}$ chromosome form temporary synaptic associations with different autosomes in early meiotic prophase I which normally can be found at pachytene-diplotene without signs of pachytene arrest. These associations are formed between the satellite DNA-enriched centomeric regions of $X$ chromosome and different autosomes but not involve the satellite DNA-poor centromeric region of $\mathrm{Y}$ chromosome. We suggest the mechanism of $X$ chromosome competitive replacement from such associations during synaptic correction. We showed that centromeric region of the $X$ chromosome remains free of $\gamma \mathrm{H} 2 \mathrm{Ax}$-dependent chromatin inactivation, while $\mathrm{Y}$ chromosome is completely inactivated. This findings highlights the predominant role of associations between satellite DNA-enriched regions of different chromosomes including X. We suppose that $\mathrm{X}$-autosome temporary associations is a manifestation of an additional synaptic disorders checkpoint. These associations are normally corrected before the late diplotene. We revealed that the intense spreading conditions applied to the spermatocytes I nuclei did not lead to destruction of stretched chromatin fibers of elongated chromocenters enriched in satellite DNA. Revealed by us tight associations between pericentromeric regions of different autosomal bivalents and $\mathrm{X}$ chromosome may represent the basis for a mechanism for maintaning the repeats stability in autosomes and $\mathrm{X}$ chromosome. The consequences of our findings are discussed.
\end{abstract}

Keywords: Prophase I of meiosis; chromatin; chromosome; synaptonemal complex; satellite DNA; chromocenter; nuclear architecture; sex chromosomes; chromatin silencing; MSCI; $\gamma \mathrm{H} 2 \mathrm{Ax}$; interactions of non-homologous chromosomes. 


\section{Introduction}

\section{1. pericentromeric satellite $D N A$.}

Pericentromeric satellite DNAs, a distinctive component of eukaryotic genomes, previously considered as "junk", over the past decades evolved to "powerful and active contributors to genomic and chromosomal evolution", providing genome regulation and stability [1]. Nevertherless, modern genome sequencing methods have a number of limitations due to the difficulties in the assembly and analysis of large blocks of tandemly repeated DNA elements in centromeric regions. Important information about size, structure and the role in the interchromosomal interactions of these DNA regions is still unstudied.

Murine pericentromeric chromatin composed of well-detailed satellite DNA families is of particular interest [2]. In mouse pericentromeric chromatin manifests as large blocks in the proximal ends of the chromosomes [3,4]. Dozens of different DNA satellites have been found in the pericentromeric regions of the Mus musculus chromosomes [5]. The two largest satellites, major (MajSat) and minor (MiSat) are located in the pericentromeric heterochromatin and represent AT-rich regions [6,7]. FISH probes targeting the monomers of MajSat and MiSat DNA repeats demonstrate strong signals in the centromeric regions of all autosomes and the X-chromosome (but not $\mathrm{Y}$-chromosome) of the Mus musculus karyotype [8,9]. According to Guenatri et al. (2004) the Minor satellite DNA is considered associated with the centromere functioning, while the major satellite DNA is a part of the heterochromatin responsible for the aggregation of various cluster-forming chromosomes [10]. In general murine telocentric chromosomal domain as a whole exhibits high sequence identity between nonhomologous chromosomes (>98\%) [11]. However, the role of major satellite DNA in cell division is still unclear [2].

\subsection{Chromocenters and ectopic contacts of pericentromeric satellite DNA in meiotic prophase I}

The studies performed on meiotic chromosomes are mainly focused on the prophase I nuclei. In particular, on the key substages associated with the homologous chromosome synapsis, DSB processing, early and late meiotic recombination events, including crossing over $[12,13,14,15,16,17]$. Centromeric regions in particular and heterochromatic regions in general, appear to cluster and decluster dynamically during the early meiotic prophase I in most organisms, suggesting an important role for these processes which, however, remains unclear $[12,18,17]$.

In early meiosis the non-homologous centromere pairing is an usual pattern, and later it is normally corrected by the homologous synapsis and assembly of bivalents $[19,20]$. This process is connected with the structure of chromosomal "bouquet", the clustering of the chromosomal telomere ends at a local territory of the inner nuclear envelope and promotes the initiation of synapsis in eukaryotes [12,21,22,23,24,25,26,17]. As a result, late zygotene - early pachytene nuclei are characterized by the finalization of homologous synapsis of chromosomes and complete correction of non-homologous synaptic associations [12].

In the pachytene, due to telomere declustering, the completely assembled bivalents demonstrate arc-shaped "trajectories" through the nuclear space [27]. Numerous cytogenetic studies performed on the nuclei passing through the presynaptic [20] postsynaptic $[15,28,29]$ stages of the meiotic prophase I describe the specific chromatin structures known as chromocenters which include pericentromeric satellite DNAs from different chromosomes [15]. Studies of the 3D reconstruction of murine synaptonemal complexes karyotype $(2 n=40)$ revealed that these associations of bivalents are random $[28,29]$.

\subsection{Pericentromeric satellite DNA and ectopic recombination}

Analysis of animal karyotypes raised a hypothesis that the sequence identity of the pericentromeric regions may be connected with the mechanism of frequent recombination 
exchanges between non-homologous chromosomes [11,30]. The question on the somatic or meiotic origin of such recombination mechanisms remains unresolved, though the data obtained on Drosophila points to the predominant role of meiosis [31]. Recombination events between the oppositely oriented repeats including the mouse MiSat DNA are also considered as prerequisites for Robertsonian translocations [11]. The authors assume a possible recombination mechanism should promote the stable evolutionary maintenance of telocentric karyotype in mouse [11]. The confirmation for the occurrence of DNA exchanges between nonhomologous chromosomes was also obtained in human and apes for tandemly repeated rDNA genes (nucleolus organizers, NOR, $28 \mathrm{~S}$ rDNA) [32]. The subsequent studies have revealed that recombination in the rDNA locus of higher eukaryotes is frequent enough to monitor changes within a few generations [33]. Studies of mouse meiotic nuclei revealed the association of NORs from different bivalents to form a common nucleolus [27], but could not provide any predictability in other associations of autosomal bivalents.

Possible mechanisms of ectopic recombination are being studied in the context of processing DNA double-strand breaks (DSB) [34,35]. DSB processing (formation and subsequent repair) was previously considered impossible in the heterochromatic regions near centromeres, however, the possibility of transient relaxation of heterochromatin and DSB processing has been described later [36]. DNA DSB repair in the pericentromeric regions of tandemly repeated DNA is particularly challenging, since the risk of aberrant recombination is increased in these regions, loss or duplication of information $[34,35,30]$. Nevertherless, unequal recombination events were suggested as a mechanism for the generation and fixation of subtly different higher-order repeats [37]. Later studies revealed that DSB processing is posssible in pericentromeric chromatin but induced DSBs and $\gamma \mathrm{H} 2 \mathrm{AX}$ (chromatin inactivation and recruitment of repair factors to damaged DNA) foci are rapidly disappear due to relocation and quick DNA repair $[35,38]$. Studies of DSB processing in heterochromatin suggest that single strand annealing (SSA) as well as non-homologous end joining (NHEJ) mechanisms are more involved in the DNA repair in pericentromeric regions, rather than homologous recombination $[39,40,36]$.

The Mus musculus karyotype, consisting only of telocentric chromosomes, is a remarkable model for studying the dynamics of chromosomal territories and interactions between chromosomes in meiosis [12]. Our work is an integration of the behavior of chromosomes during meiotic prophase I, immuno-FISH study of satellite DNA of chromocenters and the inactivation of sex chromosomes in mouse. The aims of this study were to investigate the interbivalental chromatin contacts in mouse meiotic karyotype as well as the associations between $\mathrm{X}$ chromosome and autosomal bivalents with the special focus on pericentromeric satellite DNA.

\section{Materials and Methods}

Preparation of synaptonemal complexes (SCs). Germ line cells, spermatocytes I, were obtained from 2 adult males of BALB/c and 2 adult males of CBA mice. Conventional spreads of primary spermatocytes nuclei were prepared and fixed using the technique developed by Navarro et al. (1981) [41]. Moderate and the intense spreading were implemented through the longer period of incubation (5-10 min.) of cell suspension on the surface of $0.2 \mathrm{M}$ sucrose drops and through the addition of $0.05 \%$ Triton X-100 in PBS. Poly-L-lysine-coated slides were used for all immunofluorescence studies.

Immunostaining procedure. Slides were washed with phosphate-buffered saline (PBS) and incubated with primary antibodies diluted in the antibody dilution buffer (ADB: $3 \%$ bovine serum albumin (BSA), $0.05 \%$ Triton $\mathrm{X}-100$ in PBS) at $4{ }^{\circ} \mathrm{C}$ overnight. SCs were detected using rabbit polyclonal antibodies against the SC axial element protein SYCP3 (ab15093, 1:250, Abcam, Cambridge, UK), centromeres were detected using human anti-centromere antibodies ACA (1:500; Antibodies Incorporated 15-234, Davis, CA, USA), and the inactivated chromatin of sex bivalents was stained with the mouse 
monoclonal anti- $\gamma \mathrm{H} 2 \mathrm{AX}$ antibodies (ab26350, 1:800, Abcam, Cambridge, UK). After washing, the corresponding secondary antibodies diluted in ADB were used: goat anti-rabbit Immunoglobulin (Ig) G, Alexa Fluor 555 (ab150078, 1:500, Abcam, Cambridge, UK), goat anti-rabbit Immunoglobulin (Ig) G, Alexa Fluor 488 (ab150077, 1:500, Abcam, Cambridge, UK), goat anti mouse Immunoglobulin (Ig) G, Alexa Fluor 555 (ab150118, 1:500, Abcam, Cambridge, UK), goat anti-human Alexa Fluor 488 (A-11013, 1:500, ThermoFischer). Secondary antibody incubations were performed in a humid chamber at $37^{\circ} \mathrm{C}$ for $2 \mathrm{~h}$.

FISH procedure. In situ hybridization with satellite DNA probes was performed in two rounds after immunostaining using the fluorescently labeled oligonucleotides MajSat-23bp (FAM) - 5` TTCACGTCCTAAAGTGTGTATTT and MiSat-26bp (TAMRA) - 5' AGTTACACTGAAAAACACATTCGTTG (Syntol, Moscow, Russia) following the protocol described by Stepakov et al. (2015) [42].

Microscopy. Slides were examined using the Axioimager D1 microscope (Carl Zeiss, Jena, Germany) equipped with the Axiocam HRm CCD camera (Carl Zeiss, Jena, Germany), Carl Zeiss filter sets (FS01, FS38HE, and FS43HE), and the image-processing AxioVision Release 4.6.3. software (Carl Zeiss, Jena, Germany). All preparations were mounted with the Vectashield antifade mounting medium with DAPI (Vector Laboratories, USA).

Analysis of SC preparation. Determination of meiotic prophase I stages and sex chromosomes identification were performed by the analysis of the combination of basic morphological criteria used in studies of meiotic cells [13;43;44]. Meiotic bivalents lengths were measured using ImageJ software (https://imagej.nih.gov/ij) and the numbers of autosomal bivalents associated with X-chromosome were identified. Data on nuclei with chromosomal associatons is availiable in Supplementary Materials (Figures SM1-SM4, xls-file "SC measurements"). BALB/c mouse spread nuclei preparation were used in the study, and the CBA spread nuclei were used to confirm that X-autosomal associations are usual for both inbred lines (Figures SM11, SM12).

\section{Results}

\subsection{Associations of $X$ chromosome with different autosomal bivalents.}

We studied spread preparations of mouse meiotic prophase I nuclei, i.e. total preparations of synaptonemal complexes. In $95-96 \%$ of pachytene-early diplotene nuclei the sex bivalent, $\mathrm{XY}$, normally formed specific structure, "sex body", spatially isolated from all autosomal bivalents. We have confirmed "sex body" formation using well known marker, phosphorylated form of the histone $\mathrm{H} 2 \mathrm{AX}, \gamma \mathrm{H} 2 \mathrm{AX}$ (Figure 1a) [45,46].

Nevertheless, besides the normal "isolated" location of sex bivalent from autosomal bivalents (Figure 1a) we revealed rare (5.6-6.1\%) nuclei with non-homologous end-to-end associations between $\mathrm{X}$ chromosome and autosomal bivalents (Figure $1 \mathrm{~b}$ ). Such associations mostly detected in pachytene and less often in diplotene (Supplementary Materials, Figures SM1-SM4) involve centromeric regions of $\mathrm{X}$ chromosome and centromeric region of one of lateral element of an autosomal bivalent (Figure $1 \mathrm{~b}, \mathrm{c}, \mathrm{f} ;$ Figure 2). We revealed that these associations are not bivalent-specific but instead could occur between $\mathrm{X}$ chromosome and different autosomal bivalents. In Supplementary Materials we presented detailed data of measured SC-karyotypes with bivalent numbers indicated (figure SM1-SM4, xls-file "SC measurements"). Thus, after measurements of SC-karyotypes without bivalents overlapping we found associations between $\mathrm{X}$ and almost all bivalents of mouse SC-karyotype: 1,2,3,4,5,6,7,8,9,10,11,12,13,14,15,16,18 and 19. Important additional findings are the sporadic end-to-end associations of autosomal bivalents (Figure $1 \mathrm{c}$, $\mathrm{d}-\mathrm{d}$; Figure SM1d) and the extremely rare end-to-end associations between $\mathrm{X}$ chromosome together with two autosomal bivalents. We found two nuclei with associations $\mathrm{X}+3+7$ (Figure $1 \mathrm{e}$ ) and $\mathrm{X}+4+16$ (Figure $1 \mathrm{f}$ ) (Table 1).

Notably that pericentromeric satellite DNA of $\mathrm{X}$ chromosome and satellite DNA of autosomal bivalents formed a joint chromocenter, visualised by FISH with Major Satellite 
DNA probe (Figure $1 \mathrm{~b}$ ). After a detailed analysis of preparations from two inbred mouse lines we found that $6.1 \%(\mathrm{BALB} / \mathrm{c})$ and $5.6 \%(\mathrm{CBA})$ nuclei respectively have such chromosomal associations (Table 1).

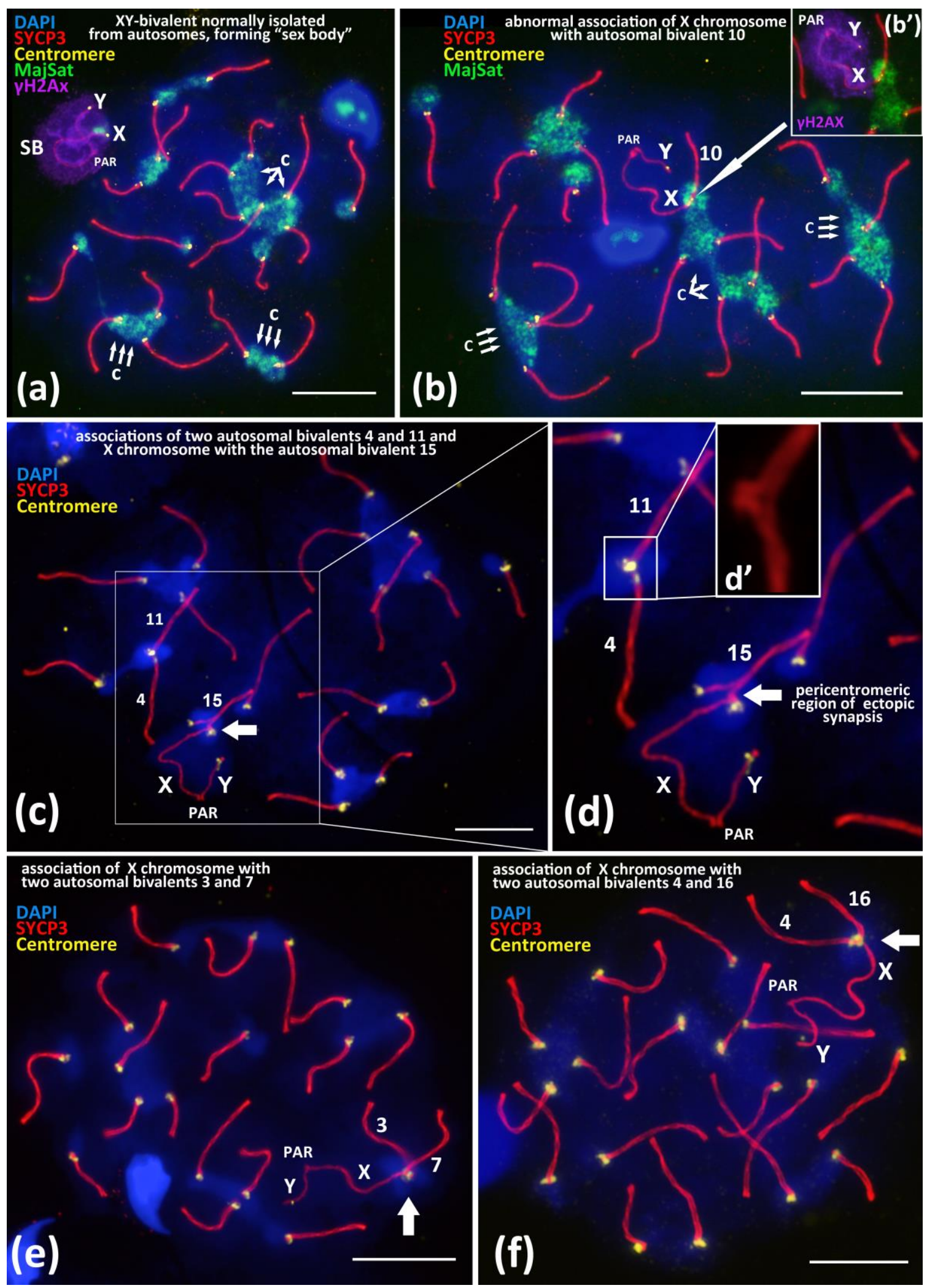

Figure 1. Associations between centromeric regions of autosomal chromosomes and $X$ chromosome in BALB/c mouse meiotic nuclei. Chromatin was stained with DAPI (blue), axial elements of meiotic chromosomes were immunostained with the antibodies against the SYCP3 protein (red), centromeres were stained with the ACA antibodies (yellow), inactivated chromatin of asynaptic sex bivalent was im- 
munostained with the antibodies against $\gamma \mathrm{H} 2 \mathrm{AX}$ (violet). FISH with oligo-DNA probes to Major satellite DNA (MajSat, green). Scale bar $-10 \mu \mathrm{m}$.

(a) - Normal meiotic behaviour of XY-bivalent forming dence DNA-protein structure "sex body" (SB), spatially isolated from autosomes in pachytene. Sex bivalent is covered by $\gamma \mathrm{H} 2 \mathrm{AX}$ immunostaining, (violet), indicating chromatin inactivation.

(b) - Association between X chromosome and autosomal bivalent 10 (white arrow). Major Satellite DNA of $X$ chromosome is included in the chromocenter (green) formed by several autosomal bivalents. (b) - immunostaining of sex bivalent with anti- $\gamma \mathrm{H} 2 \mathrm{AX}$ antibodies (violet) is located on the XY-bivalent only, but not on the assembled autosomal bivalent 10 .

(c-d) - Association of X chromosome with autosomal bivalent 15 (arrow) and (d) - details of association between two autosomal bivalents 4 and 11 .

(e) - Association of $\mathrm{X}$ chromosome with two autosomal bivalents 3 and 7. (f) - association of X- chromosome with two autosomal bivalents 4 and 16 .

Table 1. Associations between X-chromosome and autosomal bivalents in two inbred mouse strains.

\begin{tabular}{cccc}
\hline strain & $\begin{array}{c}\text { X-autosomal } \\
\text { associations N(\%) }\end{array}$ & $\begin{array}{c}\text { X-two autosomes } \\
\text { associations* }\end{array}$ & $\begin{array}{c}\text { number } \\
\text { of nuclei } \\
\text { studied }\end{array}$ \\
\hline BALB/c & $57(6.1 \%)$ & $\mathbf{N}$ & 935 \\
CBA & $48(5.6 \%)$ & 0 & 855 \\
\hline
\end{tabular}

$\mathrm{N}$ - is the number of nuclei with associations cases detected.

* - rare associations of $X$ with two autosomal bivalents preseted on Figure 1e-f.

To study the mechanism of the associations between $\mathrm{X}$ chromosome and different autosomal bivalents we traced such cases on the successive stages of meiotic prophase I which reflect the progression of chromosomal synapsis and desynapsis: late zygotene, pachytene, early- and mid-diplotene (Figure 2). Thus, in the late zygotene-early pachytene we detected the formation of short synaptonemal complex (yellow arrow) assembled side-by-side between the centromeric regions of an autosomal univalent and $X$ chromosome in the 7 nuclei (Figure 2 a). In pachytene nuclei we detected complete synapsis of all autosomal bivalents and end-to-end associations of one of these bivalents with X chromosome in 32 nuclei (Figure 2b). In early and mid-diplotene nuclei we detected partial desynapsis of autosomes (desynaptic forks), chiasmata, and end-to-end associations of $\mathrm{X}$ chromosome and partially desynapsed autosomal bivalent in 18 nuclei (Figure $2 \mathrm{c}, \mathrm{d}$ ). We also detected 6 nuclei with the disrupted associations between $X$ and autosomal bivalents in pachytene-diplotene (Supplementary Materials, Figures SM5a; SM5b). 

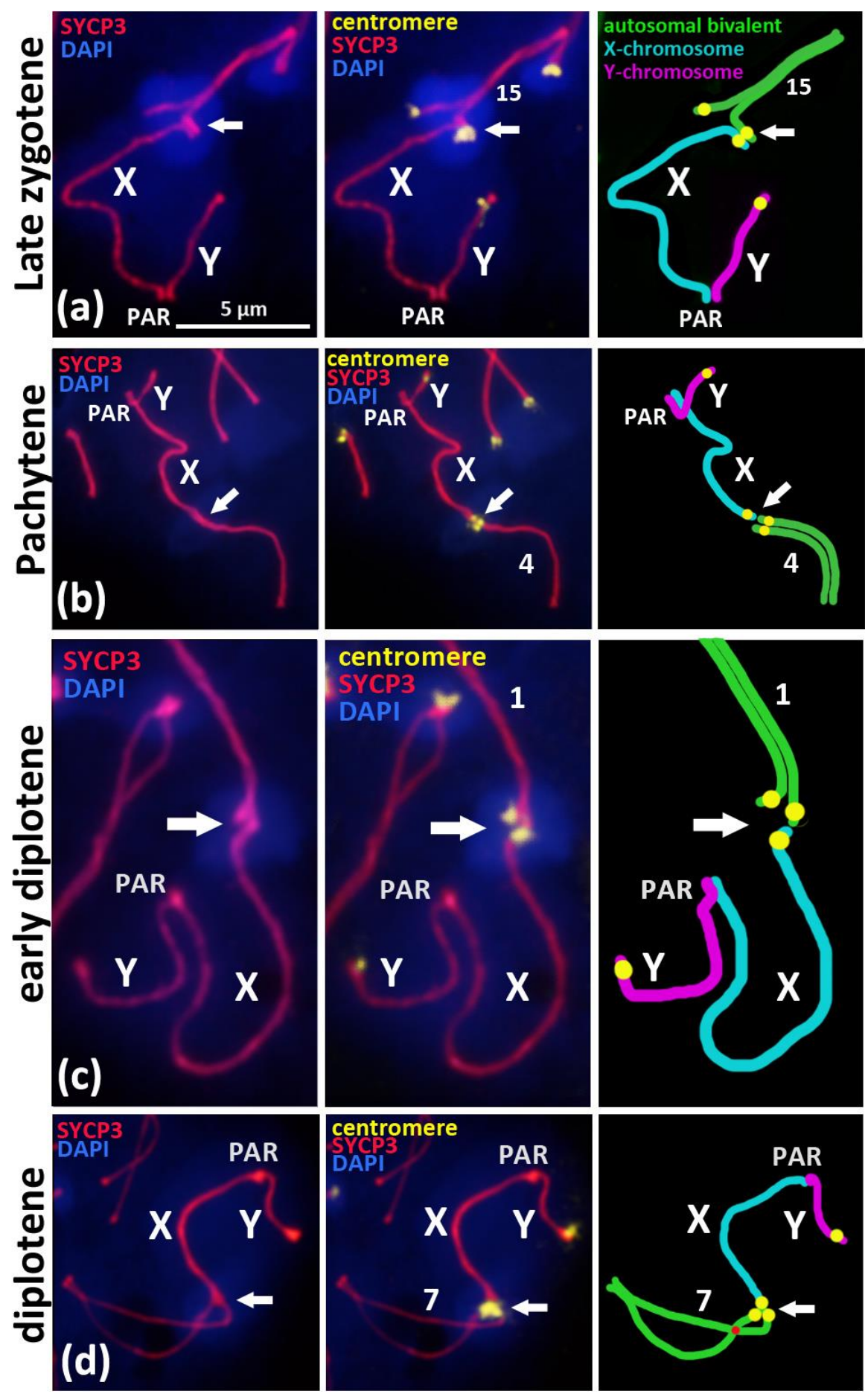

Figure 2. Associations of $\mathrm{X}$ chromosome with different autosomal bivalents at different stages of meiotic prophase I in BALB/c mouse. Chromatin was stained with DAPI (blue), axial elements of meiotic chromosomes were immunostained with the antibodies against the SYCP3 protein (red), centromeres were stained with the ACA antibodies (yellow).

(a) - Late zygotene, short synaptonemal complex (yellow arrow) assembled between the pericentromeric regions of the autosomal univalent 15 and $X$ chromosome.

(b) - Pachytene, end-to-end association of autosomal bivalent 4 and $X$ chromosome after its competitive replacement from the non-homologous SC-assembly.

(c) - Early diplotene, end-to-end association of X chromosome and autosomal bivalent 1 .

(d) - Mid-diplotene, end-to-end association of X-chromosome and autosomal bivalent 7 . 
We performed a detailed immuno-FISH study of relationships between chromocenters and $\gamma \mathrm{H} 2 \mathrm{AX}$-dependent chromatin inactivation in early zygotene and in the chromosomal associations in pachytene and diplotene (Figure 3). The study of pericentromeric regions of chromosomes at the early zygotene stage showed that all the large chromocenters (Figure 3 a-a'), which are the regions of centromeres clustering, do not undergo the explicit $\gamma \mathrm{H} 2 \mathrm{AX}$-dependent chromatin inactivation. The immunostaining of $\gamma \mathrm{H} 2 \mathrm{AX}$ is distributed over the chromatin of asynapsed chromosomes and is absent or very weak in the DAPI-rich pericentromeric chromatin (Figure 3 a-a').

Then, at the later stages of meiotic prophase I, pachytene and diplotene, we studied the areas of the $\gamma \mathrm{H} 2 \mathrm{AX}$-inactivated chromatin of the sex bivalent and the Major Satellite DNA of the proximal chromocenter which includes pericentromeric chromatin of the both $X$ chromosome and autosomal bivalent in the association. We were able to reveal that in the $X$-autosomal association, the pericentromeric region of the $X$ chromosome, enriched with MajSat DNA, was not immunostained with $\gamma \mathrm{H} 2 \mathrm{AX}$. This region is stands out from the "sex body" (Figure 3b', $c^{\prime}$ ). Additional immuno-FISH study revealed that the "sex body", immunostained with anti- $\gamma \mathrm{H} 2 \mathrm{AX}$ antibodies, and the chromocenter, which combines the pericentromeric chromatin of the $X$ chromosome and the autosomal bivalent, are clearly separated (Figure $3 \mathrm{~d}-\mathrm{d}$ ' and e-e'). That is, they represent spatially separated chromatin domains. 


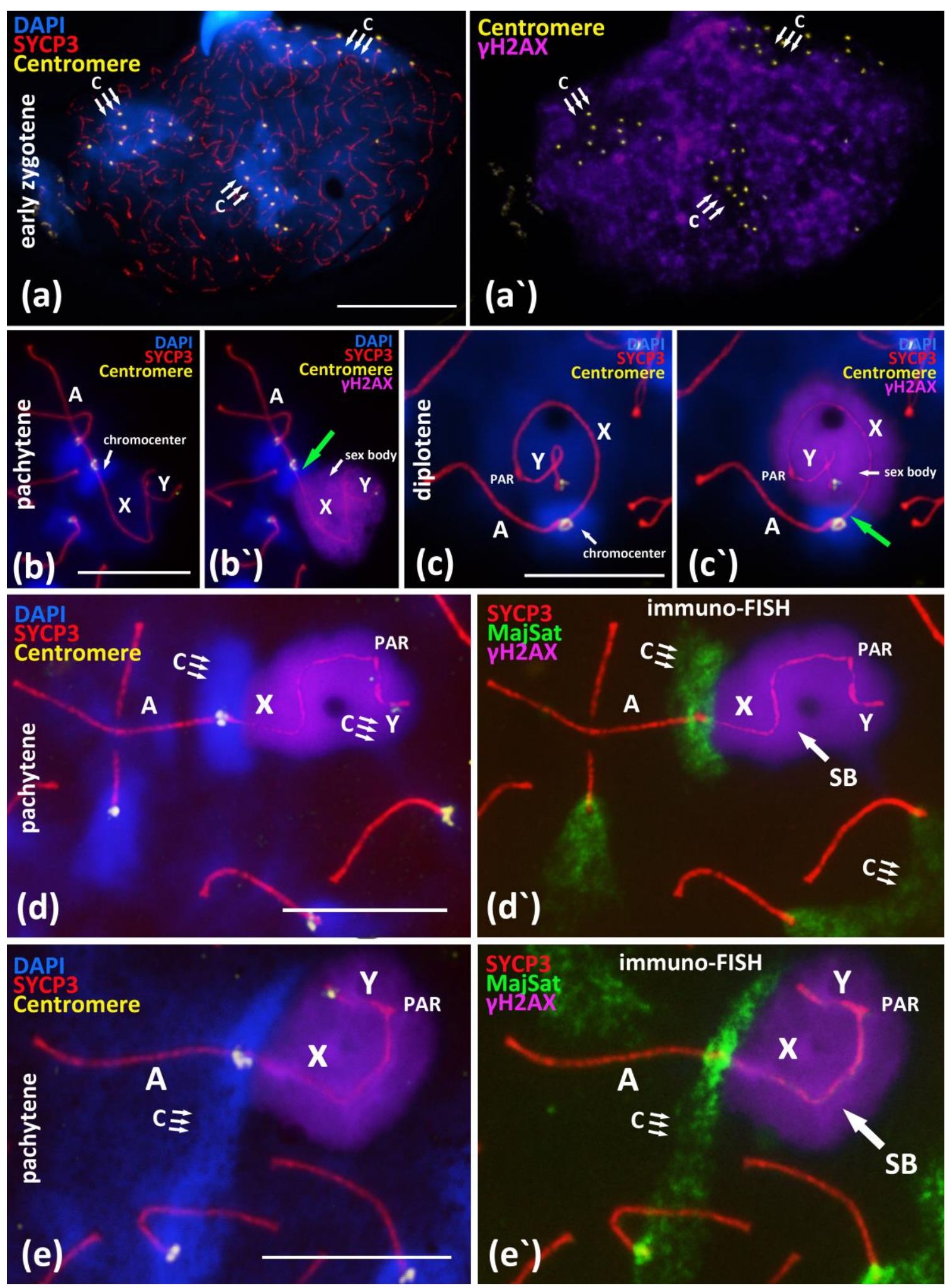

Figure 3. Immuno-FISH study of chromocenters and $\gamma \mathrm{H} 2 \mathrm{AX}$-dependent chromatin inactivation in early zygotene and in the associations between $X$ chromosome and autosomal bivalents in pachytene, BALB/c mouse. Chromatin was stained with DAPI (blue), axial elements of meiotic chromosomes were immunostained with the antibodies against the SYCP3 protein (red), centromeres were stained with the ACA antibodies (yellow), inactivated chromatin was immunostained with the antibodies against $\gamma \mathrm{H} 2 \mathrm{AX}$. FISH with oligo-DNA probes to Major Satellite DNA (MajSat, green). Scale bar $-10 \mu \mathrm{m}$.

(a) - early zygotene spread nucleus with 3 DAPI-rich chromocenters (triple arrows), composed of clustered pericentromeric regions of chromosomes; immunostained centromeres (40 yellow foci). Axial elements of asynapsed chromosomes (red). (a') - expansive $\gamma \mathrm{H} 2 \mathrm{AX}$ immunostaining on the same nucleus do not cover 3 regions of chromocenters. 
(b-c) - association of $\mathrm{X}$ chromosome and autosomal bivalent, resulted in formation of joint chromocenter. (b'-c') - immunostaining revealed that the pericentromeric region of $\mathrm{X}$ chromosome is free of $\gamma \mathrm{H} 2 \mathrm{AX}$ (green arrows) in the $\mathrm{X}$-autosomal association.

(d) - association of $X$ chromosome and autosomal bivalent, resulted in formation of the joint chromocenter (triple arrows). (d) - chromatin region of MajSat (green) and the inactivated chromatin of "sex body" (violet, $\gamma \mathrm{H} 2 \mathrm{AX}$ ), are spatially separated.

(e) - association of $X$ chromosome and autosomal bivalent, resulted in formation of joint chromocenter (triple arrows) between 2 autosomal bivalents and X chromosome. (e') - chromatin region of MajSat (green) and the inactivated chromatin of "sex body" (violet, $\gamma \mathrm{H} 2 \mathrm{AX}$ ), are spatially separated. 

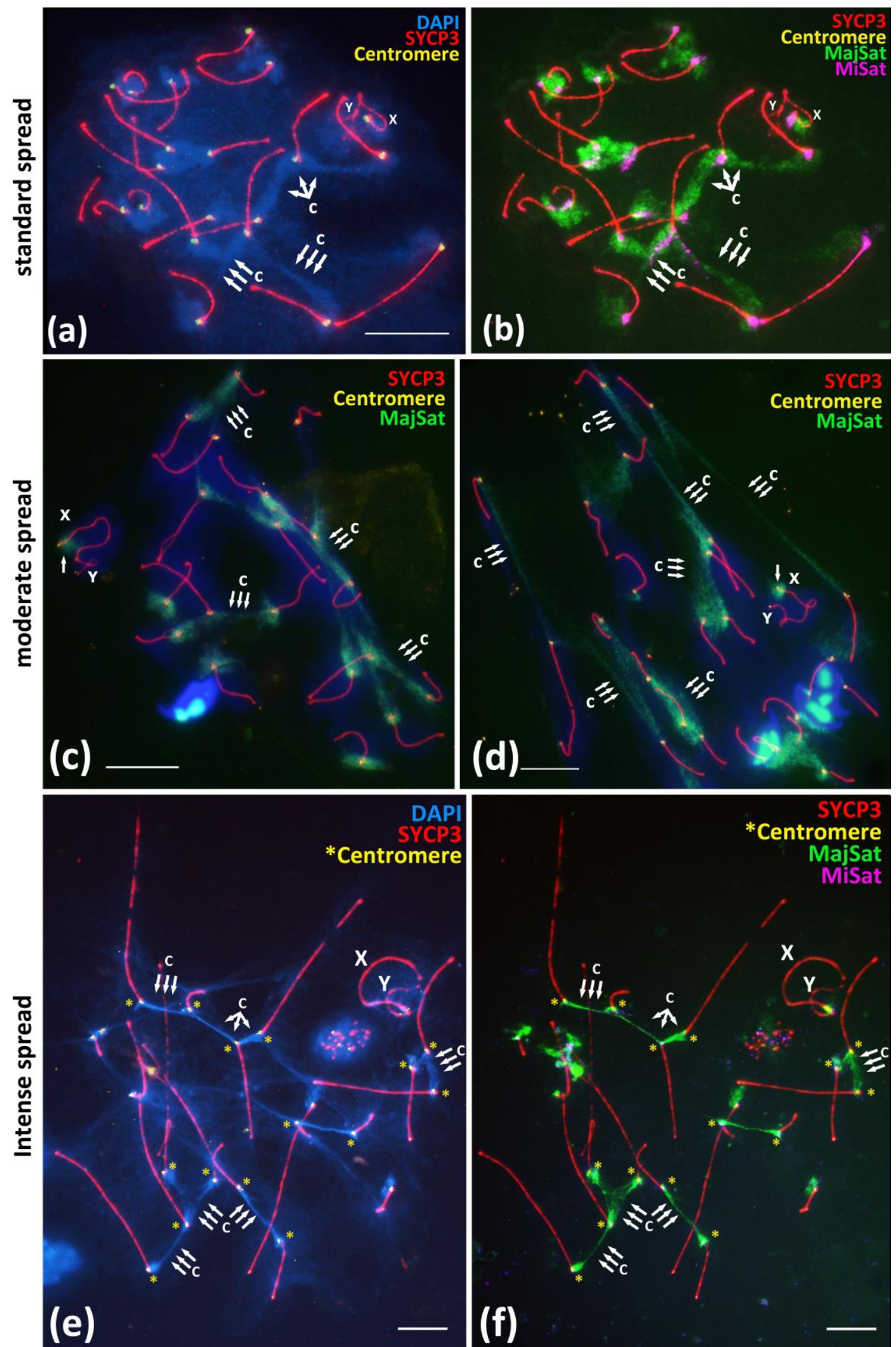

Figure 4. Immuno-FISH study of the Major and Minor satellite DNA localization in the pachytene nuclei spread preparations under different spreading conditions, BALB/c mouse. Chromatin was stained with DAPI (blue), FISH with oligo-DNA probes to Major satellite DNA (MajSat, green) and Minor satellite DNA (MiSat, violet). Axial elements of meiotic chromosomes were immunostained with the antibodies against the SYCP3 protein (red), centromeres were stained with the ACA antibodies (yellow). DAPI-enriched chromatin of chromocenters is indicated with " $\mathrm{C}^{\prime}$ and triple arrows. Sex chromosomes are indicated " $X$ " and " $Y$ ". Scale bar - $10 \mu \mathrm{m}$. 
(a-b) - Immuno-FISH on meiotic prophase I nuclei, pachytene, normal spreading. Interbivalent chromocenters demonstrate predominant coverage by Major satellite DNA (green) and smaller elongated signals of Minor satellite DNA (violet).

(c and d) - Immuno-FISH on meiotic prophase I nuclei, pachytene, moderate spreading. Chromocenters manifestate as stretched chromatin fibers enriched in MajSat DNA. Sex bivalent form spatialy separated structure and not connect with autosomal bivalents, the MajSat region of X chromosome is not stretched.

(d-e) - Immuno-FISH, pachytene, intense spreading. Tight linkage of centromeres by interbivalent stretched chromatin fibers. (d) - chromatin of chromocenters is DAPI-enriched and indicated with " $\mathrm{C}$ ". (e) - The same cell after FISH. Chromocenter chromatin fibers (green) binding the centromeric ends (yellow asterisks) of different autosomal bivalents are enriched in Major satellite DNA.

In order to analyse the morphology of satellite-DNA rich chromocenters in meitotic prophase I we performed the immuno-FISH study of the Major and Minor Satellite DNA localization in the meiotic prophase I nuclei under different spreading conditions in 114 pachytene nuclei. We obtained normal, modetate and intensive spreading of chromatin resulting in twofold spreading of pachytene nuclei (Figure 4).

Normally spreaded nuclei demonstrate the expected smooth distribution of the pachytene nuclei chromatin similar to that described in other studies $[15,27,28]$. All assembled synaptonemal complexes could be identified, and chromocenters were distinguishable as the DAPI-enriched regions between the centromeric regions of different bivalents (Figure 4 a). The immuno-FISH approach confirmed that the MajSat DNA demonstrates the predominant coverage of the chromocenters regions of autosomal bivalents and pericentromeric region of X-univalent in all 71 nuclei studied (Figure $4 \mathrm{~b}$ ). MajSat signals on the autosomal bivalents overlap the DAPI-enriched chromocenters (Figure 4 a,b, Supplementary Materials, Figures SM8 a-a', SM8 b-b '). MiSat DNA signals were located near the immunostained centromeres on all bivalents but not on the Y-chromosome similar to results of other studies [6,7,8] (Figure 4b, Supplementary Materials Figure SM7).

Moderate spreading allowed us to obtain the wider distribution of the pachytene and diplotene nuclei chromatin over the glass surface (Figure $4 \mathrm{c}$,d, Supplementary Materials, Figure SM9a-d). Immuno-FISH with the oligonucleotide probes targeted to the MajSat revealed elongation of chromocenters and forming the stretched chromatin fibers enriched in MajSat DNA. Notably, in all 23 nuclei studied no gaps of such interbivalent chromatin fibers mediated by satellite-enriched DNA were detected.

Intensely spreaded nuclei (20 nuclei studied) demonstrated not smooth, but irregular and stretched structure of the chromatin, mostly linear axial elements of SCs (bivalents) radially diverged from the center of the preparation, or stretched in one direction (Supplementary Materials, Figures SM9 e-f, SM10). DAPI-enriched chromatin territories, the chromocenters, demonstrated "cord-like" structures due to intense spreading conditions (Figure 4 e). Immuno-FISH showed that both MiSat and MajSat DNA were detected as the stretched linear interbivalent signals (Figure $4 \mathrm{f}, \mathrm{SM} 7$, SM9 e-f, SM10).

\section{Discussion}

\subsection{Chromocenters and associations between non-homologous chromosomes in meiosis}

It is known that in the early stages of meiotic prophase I (leptotene and zygotene) the formation of the specific chromosomal "bouquet" occurs, i.e. clustering of telomere ends of meiotic chromosomes $[23,26]$. Scherthan (2001) higlights that during "bouquet" formation «most terminal chromosome fractions are aligned, allowing intensive chromatin interactions» [23]. Later, at late zygotene and early pachytene telomere ends of the assembling bivalents begin to decluster and became evenly distributed over the inner nuclear envelope in mid-pachytene [23]. Thus, chromosomal "bouquet" provides the initiations of contacts of telomeric ends at leptotene, promotes the corrections of synaptic errors (non-homologous synapsis) at zygotene and results in residual associations between the bivalents on the postsynaptic stages of meiotic prophase I (Supplementary Materials Figure SM6) [12;19;20]. Previous studies of meiotic nuclei architecture in mouse revealed that after the chromosomal "bouquet" stage the declustering of the bivalent's ends leads to the formation of smaller "aggregates", chromocenters, between bivalents in late zygotene and at pachytene stages which remain stable until later stages of the meiotic prophase I $[29,20]$. Studies using mathematical models suggest random but not specific associations between bivalents in mouse 
meiotic nuclei $[27,28]$. This is consistent with our immuno-FISH data presented above (Figure $1 \mathrm{a}$-f; SM1-SM4). Both, the interbivalental connections by chromocenters as well as residual associations between centromeric regions of non-homologous chromosomes including $X$ chromosome are initiated in early meiosis by centromere clustering [20].

\subsection{Sex chromosomes behaviour and chromatin inactivation during meiotic prophase I.}

Analysis of the sex-bivalent behavior in meiosis is of particular importance. Centromeric regions of $\mathrm{X}$ and $\mathrm{Y}$ sex chromosomes do not synapse during meiotic prophase I in mouse (Figure $1 \mathrm{a}$ ) $[45,46,47]$. The only region of partial synapsis (pseudoautosomal region, PAR) on the sex bivalent is located at the distal regions of $X$ and $\mathrm{Y}$ [48]. It is also known that the XY-bivalent demonstrate the distinct behavior apart from autosomal bivalents in meiotic prophase I in mouse [45,49]. This manifests in the delay of key events: synapsis and DSB repair [50,51]. In addition, sex chromosomes are subjected to silencing during meiotic prophase I by a mechanism called meiotic sex chromosome inactivation (MSCI) leading to the formation of the "sex body", i.e. the dence DNA-protein structure, covering the sex bivalent $[45,46,49,52]$. Thus, during pachytene and diplotene stages sex bivalent demontrate the morphological features of the processes initiated earlier in leptotene-zygotene but not completed due to limited region of homology and MSCI [45,51].

\subsection{Detailing of the mechanism of associations of X-chromosome and different autosomal bivalents in meiotic prophase I.}

In our study we analysed the $\mathrm{X}$-autosomal associations on the successive stages of meiotic prophase I [Figure 1 b-f; Figure 2; Figure 3]. Late zygotene nuclei with $\mathrm{X}$-autosomal associations demonstrate the side-by-side assembly of short synaptonemal complex in pericentromeric region of $\mathrm{X}$ chromosome and asynaptic region of autosomal bivalent (Figure 2a). This important finding highlights that the initial mechanism of such associations is the meiotic synapsis - pericentromeric assembly of short SC. Normally, local regions of non-homologous synapsis should be corrected during zygotene stage and been completely corrected before pachytene starts [20]. Here we detected the delay in correction of such ectopic synaptic regions.

Later in pachytene and diplotene stages the end-to-end associations between $\mathrm{X}$ and one of the autosomal chromosomes (Figure $2 \mathrm{~b}, \mathrm{c}, \mathrm{d}$ ) are evidently the result of competitive replacement of $\mathrm{X}$ chromosome by the second homologous autosome formed the completely assembled SC (Figure 2 $\mathrm{b}, \mathrm{c}, \mathrm{d}$ ). Important confirmation of our idea is the fact that $\mathrm{X}$ chromosome is attached to only one chromosome from the ectopically-associated autosomal bivalent (Figure 2 a,b,c; SM4f). Thus, we suppose that such associations we often detect in pachytene and diplotene between $\mathrm{X}$ chromosome and autosomal bivalents are the result of "unfinished synaptic corrections" of random associations between centomeric regions of $\mathrm{X}$ and autosomes initiated in early meiosis.

It is known that non-homologous chromosomes associations normally occur in early meiosis and well detailed in animals [53;54] and plants [55]. Recently, Kasemi and Taketo (2021) revealed that in SC-karyotype of BALB/c mouse the centromeric regions remain in asynaptic state longer in late zygotene than the distal chromosome ends [17]. This is consistent with our results (Figure $1 \mathrm{c}-\mathrm{d}$ ) and could explain the preservation or formation of non-homologous synaptic associations namely between centromeric regions of autosomal chromosomes and X.

According to our results the associations between $\mathrm{X}$ chromosome and an autosomal bivalents is retained until pachytene-diplotene stages in 5.6-6.1\% of cells (Table 1). It cannot be ruled out that such associations of the $X$ chromosome with autosomes are a manifestation of the "controlling" role of the $\mathrm{X}$ chromosome. It is known that in spermatocytes of sterile males heterozygous for chromosomal translocations, disruption of chromosome synapsis and incomplete DSBs repair in meiotic prophase I leads to so called pachytene arrest of meiosis [56]. This morphologically expressed in the association of the XY-bivalent with heteromorphic SC-bivalents or SC-multivalents in which synapsis and DSBs DNA repair are impaired [52,56,57]. In this case, the sex bivalent does not migrate to the periphery of the nucleus (i.e., does not form "sex body"), but "anchors" among the autosomal 
SCs [57]. Normally in mouse pachytene arrest is observed in 3-5\% pachytene nuclei [58]. Detailed analysis of such associations resulted in the idea that non-homologous synapsis between asynaptic autosomes and $X$-chromosome is a possible way to escape the pachytene checkpoint [56,59]. Our findings of the nuclei with resolved ectopic associations between $X$ and autosomes in pachytene-diplotene indicate the possibility of correction the X-autosomal associations under study before metaphase I and thus, avoiding arrest and subsequent apoptosis of such cells (Supplementary Materials, Figures SM5a; SM5b).

Thus, we can designate the successive stages of correction of ectopic pericentromeric synapsis between $\mathrm{X}$ chromosome and autosomes (Figure $2 \mathrm{a}, \mathrm{b}, \mathrm{c}, \mathrm{d}$ ) during meiotic prophase I:

1. ectopic SC-assembly. Non-homologous local pericentromeric side-by-side synapsis of $X$ chromosome and an autosomal univalent. This stage occurs at early meiosis and charachterized by incomlete assembly of autosomal bivalent, i.e. asynaptic fork at the pericentromeric region (Figure 2 a).

2. correction of non-homologous synaptic X-autosomal association. This stage includes complete assembly of the autosomal bivalent and the competitive replacement of $X$ chromosome from the short non-homologous SC-region (Figure $3 \mathrm{~b}$-c). As a result, $\mathrm{X}$ chromosome remain associated but end-to-end with only one autosomal chromosome (Figure $3 \mathrm{~b}-\mathrm{c}$ ).

3. residual end-to-end association of $X$ chromosome with centromeric region of the assembled autosomal bivalent. Such associations can persist until diplotene (Figure 3d), and could also be disrupted before the end of prophase I (Supplementary Materials Figures SM5a; SM5b).

\subsection{Formation of X-autosomal chromocenters and MSCI.}

The most interesting question is how meiotic sex chromosomes inactivation (MSCI) act in the case of ectopic associations with autosomes. As we highlighted above, sex chromosomes in mammals normally form "sex body", the dence DNA-protein structure which should protect the sex bivalent from any type of ectopic associations $[45,49]$. Thus, in our work we demonstrated the deviations from this rule, normally occuring in $6.1 \%$ of meiotic prophase I nuclei on the postsynaptic stages in $\mathrm{BALB} / \mathrm{c}$ males (Table 1 ).

Important aspect of non-homologous chromosomal associations we describe is the contacts between pericentromeric satellite DNA of $\mathrm{X}$ chromosome and chromocenters which include pericentromeric satellite DNA of one or several autosomal bivalents (Figure 1 b; Figure 3 d',e`; Figure SM7). We detailed the involvement of pericentromeric DNA of $X$ chromosome in different autosomal chromocenters (Figure $1 \mathrm{~b}$; Figure $\left.3 \mathrm{~d}{ }^{\prime}, \mathrm{e}^{\prime}\right)$ and localized the inactivated chromatin $(\gamma \mathrm{H} 2 \mathrm{AX})$ of "sex body". Our results surprisingly showed that MajSat DNA-rich pericentromeric region of $X$ chromosome is located outside the $\gamma \mathrm{H} 2 \mathrm{AX}$ immunostaining of "sex body" (Figure $3 \mathrm{~d}$ ',e', SM7). Thus, the chromocenter which includes pericentromeric satellite DNA of autosomes and X chromosome is spatially separated from the inactivated chromatin of "sex body" despite the obligatoriness of MSCI mechanism in pachytene. This result indicates the high importance of interbivalent contacts of pericentromeric DNA in meiosis. Thus, in the hierarchy of the two processes, the non-homologous contacts of pericentromeric chromatin (chromocenters) and the MSCI, the first role goes to the chromocenters but not to MSCI. In the X-autosomal associations under study we assume the primary role of interbivalental chromocenter's formation, but not $\gamma \mathrm{H} 2 \mathrm{AX}$-dependent chromatin inactivation. In adition, MSCI is considered as the part of more general mechanism called meiotic silencing of unsynapsed chromatin (MSUC) [45]. In the pachytene nuclei we studied with complete synapsis of all autosomal bivalents and with end-to-end X-autosomal associations, the MajSat DNA of X chromosome, being the part of X-autosomal temporary chromocenter, may be under the control of autosomal MSUC mechanism but not MSCI. 
Previous studies of Major and Minor Satellite DNAs in the mouse karyotype [11] as well as our data confirm their localization in pericentromeric regions of all autosomal chromosomes and $\mathrm{X}$-chromosome, but not in the Y-chromosome (Figure $1 \mathrm{a}$,b; Figure $4 \mathrm{~b}$; Supplementary materials, Figures SM7, SM8). Thus, Y-chromosome is the only chromosome in the Mus musculus karyotype, which is isolated from the homologous meiotic synapsis in its pericentromeric region through generations. In addition, our results demonstrated that centromeric region of $Y$ chromosome which does not contains both satellite DNAs, is not involved in the chromocenters formation with autosomes in zygotene-diplotene (Figures 1-3; Supplementary Materials Figures SM1-SM4). This fact may be connected with preservation of autosome-like satellite DNA on centromeric region of $X$ chromosome but not on $\mathrm{Y}$ chromosome.

\subsection{Peculiarities of chromocenters in meiosis.}

The results we obtained using three types of spreading of meiotic nuclei, normal, moderate and the intense, demonstrate that the significant part of the "stretched chromatin fibers" connecting the centromeric regions of autosomal bivalents is occupied by the Major Satellite DNA (Figure 4). Even the intense stretching of chromatin did not lead to destruction of chromocenters (Figure $4 \mathrm{f}$; Supplementary Materials Figures SM9e-f; SM10). It is logical to assume not only colocalization but the tight contacts of satellite DNA from non-homologous chromosomes in the chromocenters. Taking together, our findings of X-autosomal interactions, absence of inactivation of MajSat DNA of $\mathrm{X}$ chromosome, associations between the pericentromeric regions of non-homologous chromosomes (i.e. formation of chromocenters) previously characterized as random $[27,28]$ and characterized by us as very tight, represent the new data in favour of the hypothesis of ectopic recombination processes in meiotic prophase I, proposed previously [11,30,31]. It was shown that suppression of DSB processing complex could promote alternative SSA, NHEJ and alt-EJ parthways $[11,36,40]$. In addition, recent studied suggest that DSB processing is possible in pericentromeric chromatin but the induced DSBs and $\gamma \mathrm{H} 2 \mathrm{AX}$ foci are rapidly disappear due to relocation and prompt repair $[35,38,40]$. This is consistent with the absence or very weak of $\gamma \mathrm{H} 2 \mathrm{AX}$ immunostaining in the chromocenters in early zygotene we detected (Figure 3a-a'). Below we list our results in context of results of other authors in favor of the hypothesis [11,30,31].

- Namely random interbivalent contacts proved previously $[27,28]$ and an ectopic recombination mechanisms $[11,30]$ could allow to maintain the uniformity of satellite DNA composition between autosomal chromosomes [11] and $X$ chromosome (Figure 1 b; Figure 2; Figure 3 b-e ; Supplementary materials, Figures SM7, SM8 b,c).

- We have revealed that pericentromeric chromatin of the $X$ chromosome may be involved in ectopic interactions with chromocenters of different autosomes (18 of 19 bivalents are detected in such associations in our study) (Figure 1 b-f; Figure 2; SM1-SM5). This could the basis for a mechanism of maintaining autosome-like DNA-repeats composition near centromere of $\mathrm{X}$ chromosome.

- We have shown that the pericentromeric chromatin of the $X$ chromosome is spatially separated from the inactivated chromatin of "sex body" (Figure 1b; Figure 3 d',e'; SM8). Thus, XY-chromatin inactivation (MSCI) do not interfere with ectopic X-autosomal interactions of pericentromeric DNAs. This result indicates the high importance of chromocenters formation and functioning during meiosis.

- We have shown that twofold stretching of the meiotic nuclei chromatin on the glass surface does not destroy elongated chromocenters (Figure 4a-f; SM9-10). These "interbivalent chromatin fibers" contain highly repeated MajSat and MiSat DNA of pericentromeric regions of non-homologous chromosomes (Figure 4, SM7, SM9, SM10). This is consistent with the data that centromere but not telomere clustering is the general and primordial meiotic mechanism in eukaryotes [26].

Thus, interbivalental associations mediated by chromocenters are apparently an important mechanism for maintaining the stability of the pericenromeric satellite DNA composition. The Major Satellite, in our opinion, plays an important role in this process in mouse. The important and still un- 
resolved question is whether interchromosomal connections of satellite DNA are temporary, i.e. stage-specific and could be formed several times during cell cycle or they are constitutive, but not easily detected at certain stages. Indeed, chromatin "bridges" between chromosomes were observed in the interphase and even in mitotic metaphase plates preparations, but still have not been generalized for the entire cell cycle $[60,61,62]$.

\section{Conclusions}

1. We revealed the mechanism of associations between the satellite DNA-enriched regions of autosomes and centromeric region of $\mathrm{X}$ chromosome. We detailed the successive stages of correction of these non-homologous X-autosomal associations: early meiotic ectopic SC-assmebly followed by the competitive replacemnet of $\mathrm{X}$ chromosome by autosome in later stages [Figure $2 \mathrm{a}-\mathrm{d}$ ].

2. In the case of the $X$-autosomal association the pericentromeric region of the $X$ chromosome is integrated in the autosomal chromocenters enriched in MajSat DNA. Centromeric region of the $X$ chromosome is free of $\gamma \mathrm{H} 2 \mathrm{Ax}$-dependent chromatin inactivation (MSCI). Thus, the "Sex body" and the proximal X-autosomal MajSat-rich chromocenter are spatially and functionally separated.

3. Our results demonstrate the stability and remarkable sturdiness of the interbivalent chromatin fibers connecting centromeric regions and enriched in satellite DNA in the meiotic prophase I nuclei.

Stable interbivalental associations detailed by us, absence of chromatin inactivation in the $\mathrm{X}$-autosomal chromocenters, and the known data on the DSB processing pathways in heterochromatin [38,35] represent the new data in favour of a mechanism of maintenance of pericentromeric repeat stability in autosomes and $X[30]$ as well as for insights into the independent evolution of centromeric DNA content in autosomes and X chromosome on one hand and in $\mathrm{Y}$ chromosome - on the another.

Supplementary Materials: Figures SM1-SM4: «Associations between centromeric regions of autosomal chromosomes and $\mathrm{X}$ chromosome in BALB/c mouse meiotic nuclei», Figure SM5: «Disrupted associations between centromeric regions of autosomal chromosomes and $\mathrm{X}$ chromosome in $\mathrm{BALB} / \mathrm{c}$ mouse meiotic nuclei in pachytene and diplotene», Figure SM6: «Chromocenters in early zygotene and pachytene in BALB/c mouse», Figure SM7: «Immuno-FISH with oligo-DNA probes to Minor satellite DNA in the spread preparation of meiotic nucleus BALB/c mouse», Figure SM8: «Immuno-FISH identification of MSCI in the associations between Major satellite DNA of X chromosome and autosomal chromocenters», Figures SM9-SM10: «Immuno-FISH study of the Major satellite DNA localization in the pachytene nuclei spread preparations under different spreading conditions, BALB/c mouse», Figures SM11-SM12: «Associations between centromeric regions of autosomal chromosomes and $\mathrm{X}$ chromosome in CBA mouse meiotic nuclei».

Author Contributions: Conceptualization, V.S. and O.K.; methodology, V.S., M.L., I.V. and P.N.; resources, S.S., validation, V.S. and O.K.; formal analysis, V.S; writing - original draft preparation, V.S.; writing - review and editing, V.S.; visualization, V.S.; supervision, V.S.; project administration, O.K.; funding acquisition, O.K. All authors have read and agreed to the published version of the manuscript.

Funding: The reported study was funded by the State Contract of VIGG RAS.

Institutional Review Board Statement: All experimental protocols were approved by the Ethics Committee for Animal Research of the Vavilov Institute of General Genetics (protocol No. 3, November 10, 2016) in accordance with the Regulations for Laboratory Practice.

Acknowledgments: The authors would like to thank prof. Yu. Bogdanov, Igor Mazheika, Alexandra Bogomazova, Vitaly Korchagin, Anna Novokreshchenova, Tatiana Grishaeva, Vladimir Popov. We thank the Genetic Polymorphism Core Facility of the Vavilov Institute of General Genetics of the Russian Academy of Sciences, Moscow.

Conflicts of Interest: The authors declare no conflict of interest

\section{References}


1. Hartley, G.; O'Neill, R. Centromere Repeats: Hidden Gems of the Genome. Genes 2019, 10, 3, 223, doi:10.3390/genes10030223.

2. Pajpach, F.; Wu, T.; Shearwin-Whyatt, L.; Jones, K.; Grützner, F. Flavors of Non-Random Meiotic Segregation of Autosomes and Sex Chromosomes. Genes 2021, 12, 9, 1338, doi:10.3390/genes12091338.

3. Wijchers, P.J.; Geeven, G.; Eyres, M.; Bergsma, A.J.; Janssen, M.; Verstegen, M.; De Laat, W. Characterization and dynamics of pericentromere-associated domains in mice. Genome research 2015, 25, 7, 958-969, doi:10.1101/gr.186643.114.

4. Pardue, M.L.; Gall, J.G. Chromosomal localization of mouse satellite DNA. Science 1970, 168, 3937, 1356-1358, doi:10.1126/science.168.3937.1356.

5. Podgornaya, O.I.; Ostromyshenskii, D.I.; Enukashvily, N.I. Who needs this junk, or genomic dark matter. Biochemistry (Moscow) 2008, 83, 4, 450-466, doi:10.1134/S0006297918040156.

6. Kipling, D.; Wilson, H. E.; Mitchell, A. R.; Taylor, B. A.; Cooke, H. J. Mouse centromere mapping using oligonucleotide probes that detect variants of the minor satellite. Chromosoma 1994, 103, 1, 46-55, doi:10.1007/BF00364725.

7. Komissarov, A.S.; Gavrilova, E.V.; Demin, S.J.; Ishov, A.M.; Podgornaya, O.I. Tandemly repeated DNA families in the mouse genome. BMC genomics 2011, 12, 1, 531, doi:10.1186/1471-2164-12-531.

8. Wong, A.K.C.; Biddle, F.G.; Rattner, J.B. The chromosomal distribution of the major and minor satellite is not conserved in the genus Mus. Chromosoma 1990, 99, 3, 190-195, doi:10.1007/BF01731129.

9. Garagna, S.; Redi, C.A.; Capanna, E.; Andayani, N.; Alfano, R.M.; Doi, P.; Viale, G. Genome distribution, chromosomal allocation and organization of the major and minor satellite DNA in 11 species and subspecies of the genus Mus. Cytogenetic and Genome Research 1993, 64, 3-4, 247-255, doi:10.1159/000133587.

10. Guenatri, M.; Bailly, D.; Maison, C.; Almouzni, G. Mouse centric and pericentric satellite repeats form distinct functional heterochromatin. Journal of Cell Biology 2004, 166, 4, 493-505, doi:10.1083/jcb.200403109.

11. Kalitisis, P.; Griffiths, B.; Choo, K.A. Mouse telocentric sequences reveal a high rate of homogenization and possible role in Robertsonian translocation. Proceedings of the National Academy of Sciences 2006, 103, 23, 8786-8791, doi:10.1073/pnas.0600250103.

12. Scherthan, H.; Weich, S.; Schwegler, H.; Heyting, C.; Härle, M.; Cremer, T. Centromere and telomere movements during early meiotic prophase of mouse and man are associated with the onset of chromosome pairing. J Cell Biol. 1996, 134, 5, 1109-25, doi:10.1083/jcb.134.5.1109.

13. Zickler, D.; Kleckner, N. Meiotic chromosomes: integrating structure and function. Annual review of genetics 1999, 33, 1, 603-754, doi:10.1146/annurev.genet.33.1.603.

14. Zickler, D.; Kleckner, N. A few of our favorite things: Pairing, the bouquet, crossover interference and evolution of meiosis. In Seminars in cell \& developmental biology 2016, 54, 135-148, doi:

10.1016/j.semcdb.2016.02.024.

15. Berrios, S. Nuclear architecture of mouse spermatocytes: chromosome topology, heterochromatin, and nucleolus. Cytogenetic and genome research 2017, 151, 2, 61-71, doi:10.1159/000460811.

16. Ur, S.N.; Corbett, K.D. Architecture and Dynamics of Meiotic Chromosomes. Annual Review of Genetics 2021, 55. doi:10.1146/annurev-genet-071719-020235.

17. Kazemi, P.; Taketo, T. Two telomeric ends of acrocentric chromosome play distinct roles in homologous chromosome synapsis in the fetal mouse oocyte. Chromosoma 2021, 130, 1, 41-52, doi: 10.1007/s00412-021-00752-1.

18. Koszul, R.; Kleckner, N. Dynamic chromosome movements during meiosis: a way to eliminate unwanted connections? Trends in cell biology 2009, 19, 12, 716-724, doi:10.1016/j.tcb.2009.09.007.

19. Stewart, M.N.; Dawson, D.S. Changing partners: moving from non-homologous to homologous centromere pairing in meiosis. Trends in Genetics 2008, 24, 11, 564-573, doi:10.1016/j.tig.2008.08.006.

20. Eyster, C.; Chuong, H.H.; Lee, C.Y.; Pezza, R.J.; Dawson, D. The pericentromeric heterochromatin of homologous chromosomes remains associated after centromere pairing dissolves in mouse spermatocyte meiosis. Chromosoma 2019, 128, 3, 355-367, doi:10.1007/s00412-019-00708-6.

21. Klutstein, M.; Cooper, J. P.. The Chromosomal Courtship Dance-homolog pairing in early meiosis. Current Opinion in Cell Biology 2014, 26, 123-131. doi:10.1016/j.ceb.2013.12.004. 
22. Scherthan, H. Telomere attachment and clustering during meiosis. Cell Mol Life Sci 2007, 64, 117-124, doi:10.1007/s00018-006-6463-2.

23. Scherthan, H. A bouquet makes ends meet. Nature reviews Molecular cell biology 2001, 2, 8, 621-627, doi:10.1038/35085086.

24. Harper, L.; Golubovskaya, I.; Cande, W.Z. A bouquet of chromosomes. Journal of Cell Science 2004, 117,18, 4025-4032, doi:10.1242/jcs.01363.

25. Zickler, D.; Kleckner, N. The leptotene-zygotene transition of meiosis. Annual review of genetics 1998, 32 , 1, 619-697, doi:10.1146/annurev.genet.32.1.619.

26. Tian, M.; Agreiter, C.; Loidl, J. Spatial constraints on chromosomes are instrumental to meiotic pairing. Journal of cell science 2020, 133, 22, jcs253724, doi:10.1242/jcs.253724

27. Berrios, S.; Manieu, C.; López-Fenner, J.; Ayarza, E.; Page, J.; González, M.; Fernández-Donoso, R. Robertsonian chromosomes and the nuclear architecture of mouse meiotic prophase spermatocytes. Biological research 2014, 47,16, doi:10.1186/0717-6287-47-16.

28. López-Fenner, J.; Berríos, S.; Manieu, C.; Page, J.; Fernández-Donoso, R. Bivalent Associations in Mus domesticus 2n=40 Spermatocytes. Are They Random? Bulletin of mathematical biology 2014, 76, 8, 1941-1952, doi:10.1007/s11538-014-9992-0.

29. Berríos, S.; Manterola, M.; Prieto, Z.; López-Fenner, J.; Page, J.; Fernández-Donoso, R. Model of chromosome associations in Mus domesticus spermatocytes. Biological research 2010, 43, 3, 275-285, doi:10.4067/S0716-97602010000300003.

30. Peng, J.C.; Karpen, G.H. Epigenetic regulation of heterochromatic DNA stability. Current opinion in genetics $\mathcal{E}$ development 2008, 18(2), 204-211, doi:10.1016/j.gde.2008.01.021.

31. Montgomery, E.A.; Huang, S.M.; Langley, C.H.; Judd, B.H. Chromosome rearrangement by ectopic recombination in Drosophila melanogaster: genome structure and evolution. Genetics 1991, 129, 4, 1085-1098, doi:10.1093/genetics/129.4.1085.

32. Arnheim, N.; Krystal, M.; Schmickel, R.; Wilson, G.; Ryder, O.; Zimmer, E. Molecular evidence for genetic exchanges among ribosomal genes on nonhomologous chromosomes in man and apes. Proceedings of the National Academy of Sciences 1980, 77, 12, 7323-7327, doi:10.1073/pnas.77.12.7323.

33. Eickbush, T.H.; Eickbush, D.G. Finely orchestrated movements: evolution of the ribosomal RNA genes. Genetics 2007, 175, 2, 477-485, doi:10.1534/genetics.107.071399.

34. Amaral, N.; Ryu, T.; Li, X. and Chiolo, I. Nuclear dynamics of heterochromatin repair. Trends in Genetics 2017, 33, 2, 86-100, doi: 10.1016/j.tig.2016.12.004.

35. Chiolo, I.; Minoda, A.; Colmenares, S. U.; Polyzos, A.; Costes, S. V.; Karpen, G. H. Double-strand breaks in heterochromatin move outside of a dynamic HP1a domain to complete recombinational repair. Cell 2011, 144(5), 732-744, doi:10.1016/j.cell.2011.02.012.

36. Goodarzi, A.A.; Jeggo, P.A. The heterochromatic barrier to DNA double strand break repair: how to get the entry visa. International journal of molecular sciences 2012, 13, 9, 11844-11860, DOI: 10.3390/ijms130911844.

37. Waye, J.S.; Willard, H.F. Molecular analysis of a deletion polymorphism in alpha satellite of human chromosome 17: evidence for homologous unequal crossing-over and subsequent fixation. Nucleic acids research 1986, 14, 17, 6915-6927, DOI: 10.1093/nar/14.17.6915.

38. Jakob, B.; Splinter, J.; Conrad, S.; Voss, K. O.; Zink, D.; Durante, M.; Taucher-Scholz, G. DNA double-strand breaks in heterochromatin elicit fast repair protein recruitment, histone H2AX phosphorylation and relocation to euchromatin. Nucleic acids research 2011,39, 15, 6489-6499, DOI:10.1093/nar/gkr230.

39. Lemaitre, C.; Soutoglou, E. Double strand break (DSB) repair in heterochromatin and heterochromatin proteins in DSB repair. DNA repair 2014, 19, 163-168, doi: 10.1016/j.dnarep.2014.03.015.

40. Tsouroula, K.; Furst, A.; Rogier, M.; Heyer, V.; Maglott-Roth, A.; Ferrand, A.; Reina-San-Martin B.; Soutoglou, E. Temporal and spatial uncoupling of DNA double strand break repair pathways within mammalian heterochromatin. Molecular cell 2016, 63, 2, 293-305, DOI: 10.1016/j.molcel.2016.06.002.

41. Navarro, J.; Vidal, F.; Guitart, M.; Egozcue, J. A method for the sequential study of synaptonemal complexes by light and electron microscopy. Human Genetics 1981, 59, 4, 419-421, doi: 10.1016/s0304-4017(00)00412-x. 
42. Stepakov, A.; Galkina, S.; Bogomaz, D.; Gaginskaya, E.; Saifitdinova, A. Modified synthesis of 6-carboxyfluorescein (6-FAM): application to probe labeling for conventional cytogenetics. Current Journal of Applied Science and Technology 2015, 7, 4, 423-428, DOI:10.9734/BJAST/2015/15991

43. Bogdanov, Y.F.; Kolomiets, O.L. Synaptonemal complex as an indicator of the dynamics of meiosis and chromosome variation. 2007. Moscow, KMK Press, 359.

44. Spangenberg, V.; Arakelyan, M.; Galoyan, E.; Pankin, M.; Petrosyan, R.; Stepanyan, I.; Grishaeva, T.; Danielyan, F.; Kolomiets, O. Extraordinary centromeres: differences in the meiotic chromosomes of two rock lizards species Darevskia portschinskii and Darevskia raddei. PeerJ 2019, 7:e6360, DOI: 10.7717/peerj.6360.

45. Turner, J.M. Meiotic sex chromosome inactivation. Development 2007, 134, 10, 1823-1831, doi: 10.1242/dev.000018.

46. Turner, J.M. Meiotic silencing in mammals. Annual review of genetics 2015, 49, 395-412, doi: 10.1146/annurev-genet-112414-055145.

47. Burgoyne, P.S. Genetic homology and crossing over in the $X$ and $Y$ chromosomes of mammals. Hum Genet 1982, 61, 85-90, doi: 10.1007/BF00274192.

48. Qiao, H.; Chen, J.K.; Reynolds, A.; Hoog, C.; Paddy, M.; Hunter, N. Interplay between synaptonemal complex, homologous recombination and centromeres during mammalian meiosis. PLoS Genet. 2012, 8, 6, e1002790, DOI:10.1371/journal.pgen.1002790.

49. Solari, A.J. The behaviour of the XY pair in mammals. Int. Rev. Cytol. 1974, 38, 273-317, doi: 10.1016/s0074-7696(08)60928-6.

50. Sciurano, R.B.; Solari, A.J. Ultrastructural and Immunofluorescent Methods for the Study of the XY Body as a Biomarker. Methods Mol Biol. 2014, 1094:137-49, doi: 10.1007/978-1-62703-706-8_11.

51. Waters, P.D.; Ruiz-Herrera, A. Meiotic executioner genes protect the $\mathrm{Y}$ from extinction. Trends in Genetics, 2020, 36, 10, 728-738, doi: 10.1016/j.tig.2020.06.008

52. Manterola, M.; Page, J.; Vasco, C.; Berríos, S.; Parra, M. T.; Viera, A.; , Rufas, J.; Zuccotti, M.; Garagna, S.; Fernández-Donoso, R. A high incidence of meiotic silencing of unsynapsed chromatin is not associated with substantial pachytene loss in heterozygous male mice carrying multiple simple robertsonian translocations. PLoS genetics 2009, 5, 8, e1000625, DOI: 10.1371/journal.pgen.1000625.

53. Rasmussen, S.W.; Holm, P.B. Chromosome pairing in autotetraploid Bombyx females. Mechanism for exclusive bivalent formation. Carlsberg Research Communications 1979, 44, 2, 101, doi:10.1007/BF02906525.

54. Woglar, A. and Jantsch, V. Chromosome movement in meiosis I prophase of Caenorhabditis elegans. Chromosoma 2014, 123, 15-24, doi: 10.1007/s00412-013-0436-7.

55. Pawlowski, W.P. Chromosome organization and dynamics in plants. Curr. Opin. Plant Biol. 2010, 13, 640-645, DOI: 10.1016/j.pbi.2010.09.015.

56. Oliver-Bonet, M.; Ko, E.; Martin, R.H. Male infertility in reciprocal translocation carriers: the sex body affair. Cytogenetic and genome research 2005, 111, 3-4, 343-346, DOI: 10.1159/000086908.

57. Homolka, D.; Ivanek, R.; Capkova, J.; Jansa, P.; Forejt, J. Chromosomal rearrangement interferes with meiotic X chromosome inactivation. Genome Research 2007, 17, 10, 1431-1437, DOI: 10.1101/gr.6520107.

58. Kolomiets, O. L.; Abuduev, N. K.; Mazurova, T. F.; Bragina, E. E.; Dadashev S. Ya.; Kurilo, L.F.; Bogdanov, Y. F. Damaging effect of antibiotics on the structure of synaptonemal complexes of meiotic mouse chromosomes. Russian Journal of Genetics 2001, 37, 2, 141-149.

59. Voet, T.; Liebe, B.; Labaere, C.; Marynen, P.; Scherthan, H. Telomere-independent homologue pairing and checkpoint escape of accessory ring chromosomes in male mouse meiosis. The Journal of cell biology 2003, 162, 5, 795-808, doi:10.1083/jcb.200305065.

60. Saifitdinova, A.F.; Timofejeva, L.P.; Zhurov, V.G.; Gaginskaya, E.R. A highly repeated FCP centromeric sequence from chaffinch (Fringilla coelebs: Aves) genome is revealed within interchromosomal connectives during mitosis. Tsitologiia 2000, 42, 6, 581-586.

61. Saifitdinova, A.F.; Derjusheva, S.E.; Malykh, A.G.; Zhurov, V.G.; Andreeva, T.F.; Gaginskaya, E.R. Centromeric tandem repeat from the chaffinch genome: isolation and molecular characterization. Genome 2001, 44, 1, 96-103, DOI:10.1139/gen-44-1-96.

62. Kuznetsova, I.S.; Enukashvily, N.I.; Noniashvili, E.M.; Shatrova, A.N.; Aksenov, N.D.; Zenin, V.V.; Podgornaya, O.I. Evidence for the existence of satellite DNA-containing connection between metaphase chromosomes. Journal of cellular biochemistry 2007, 101, 4, 1046-1061. 
